\title{
Endoscopy and the Risk of Venous Thromboembolism: A Case-Control Study
}

Authors

Institutions
S. V. Venkatachalapathy ${ }^{1}$, G. Evans ${ }^{2}$, A. F. Muller ${ }^{1}$

${ }^{1}$ Department of Gastroenterology, The Kent and Canterbury Hospital, Canterbury, Kent, United Kingdom

${ }^{2}$ Department of Haematology, The Kent and Canterbury Hospital, Canterbury, Kent, United Kingdom received

15. November 2013 accepted after revision 20. January 2014

\section{Bibliography}

DOI http://dx.doi.org/

10.1055/s-0034-1365277

Published online: 10.3.2014

Endoscopy International Open

2014; 02: E2-E5

(c) Georg Thieme Verlag KG

Stuttgart · New York

E-ISSN 2196-9736

\section{Corresponding author}

Dr. A.F. Muller, DM, FRCP

Department of

Gastroenterology

The Kent and Canterbury

Hospital

Ethelbert Road, Canterbury,

Kent, CT1 3NG

United Kingdom

andrew.muller@nhs.net
Background and Study Aims To assess whether there was an association between endoscopy and the risk of venous thromboembolism (VTE).

Patients and Methods Retrospective case - control study of patients diagnosed with VTE over a 3year period. Each was age- and sex-matched to one of three controls who attended an outpatient appointment on the same date as that of the diagnosis of VTE in the patients. Patients who had undergone endoscopy within 90 days of VTE were included. On a second analysis, patients who were hospitalized and those with inflammatory bowel disease or malignancy were excluded. The difference in occurrence of endoscopy between cases and controls was examined using the McNemar test. The risk of VTE occurring following endoscopy was quantified by means of odds ratios.

Results Forty-five of 436 patients (10.3\%) had undergone an endoscopy in the VTE group compar-

\section{Introduction}

$\nabla$

Venous thromboembolism (VTE) is the one of the commonest causes of inpatient mortality, responsible for up to 370000 deaths per annum in Europe. [1] It contributes to longer duration of hospitalization, morbidity, and mortality, with pulmonary embolism accounting for $5 \%-10 \%$ of deaths in hospitalized patients. [2] There are several risk factors for developing VTE in hospitalized patients, including surgery, intensive care unit admission, complete immobilization, and cancer. Little has been published on the risk of patients attending for endoscopy - the majority of whom are outpatients.

Many endoscopic procedures require at least 6 hours fasting, and colonoscopy needs rigorous bowel preparation. These factors may contribute to dehydration and potentially increase the risk of VTE. Patients may have to lie in the same position for a prolonged period, especially if the pro- ed with $14 / 436$ controls (3.2\%; $P<0.001)$. The odds ratio for developing a VTE after an endoscopic procedure was 3.58 (95\% CI 1.86-7.46) for patients relative to controls. When the 10 hospitalized patients and respective controls were excluded, the odds of VTE remained nearly 3 times as large for patients undergoing endoscopy as for controls (2.92 [95\% CI 1.51, 5.62]; $P=0.001$ ). When patients with inflammatory bowel disease or malignancy were also excluded, no difference was found between patients undergoing endoscopy and controls (1.92 [0.95, 3.85]; $P=0.07)$. Ten percent of patients with VTE underwent endoscopy in the 3 months before the diagnosis compared with $3 \%$ of controls $(P<0.001)$. No significant difference was found between the type of endoscopy performed and VTE risk.

Conclusions When those with known risk factors for VTE were excluded, no significant increased risk of VTE was found.

cedure involves delivering therapy. They may be confined to bed for 1-2 hours after the procedure. In addition, some patients with gastrointestinal disease appear to have an increased risk of VTE, including those with inflammatory bowel disease and cancer. $[3,4]$ The combination of dehydration, immobility, sedation, and co-morbidities may increase the risk of VTE.

This case-control study was designed to assess whether an association could be found between patients attending for endoscopic procedures and an increased risk of VTE. A secondary objective was to see whether the risk of developing VTE was associated with any particular endoscopic procedure more than others.
License terms

(๑) $\odot \circledast$ 


\section{Patients and Methods}

\section{$\nabla$}

\section{Study Design, Patient Population,} and Outcome Measures

This retrospective case - control study was conducted at the East Kent University Hospitals NHS Foundation Trust, United Kingdom. The Trust comprises three acute hospitals covering a population of 750 000.It performs around 15000 endoscopic procedures every year.

Venous thromboembolism (VTE) is defined as a disease that includes both deep vein thrombosis (DVT) and pulmonary embolism. For the purpose of this study we defined endoscopy as comprising esophagogastroduodenoscopy, flexible sigmoidoscopy, endoscopic retrograde cholangiopancreatography (ERCP), and colonoscopy.

We included all patients listed as having had a VTE on the Dawn anticoagulant monitoring system at the Kent Haemophilia Centre. The data were collected from January 2009 to December 2011. The diagnosis of VTE in the patient cohort was confirmed by a review of both $\mathrm{D}$-dimer and radiology results (Doppler ultrasound, $\mathrm{V} / \mathrm{Q}$ scanning, or computed tomography of the pulmonary arteries). Controls were identified from the East Kent University Hospitals outpatients registry. For each patient with VTE, three age- and sex-matched controls were identified who had attended outpatients on the same date as that on which the patient had been diagnosed to have VTE, and one of these was randomly selected as the control for that patient.

The hospital and endoscopy records of cases and controls were reviewed to identify those patients who had undergone endoscopy in the 3 months preceding the VTE diagnosis. This timescale was chosen based on published guidance from the UK Department of Health linking the presence of VTE to a hospital event. $[5,6]$ The difference between the VTE and the control group in terms of occurrence of endoscopy was calculated. The risk of VTE occurring following endoscopy was quantified using odds ratios.

Patients aged 18 years or older were included in the study. Those requiring endoscopic ultrasound examinations, who are referred to a neighboring hospital trust, were excluded from the analysis. The analysis compared the risk of VTE in patients attending for endoscopy compared with controls. A second analysis excluded patients with known risk factors for VTE: those with a previous diagnosis of VTE; those known to have or diagnosed at the time of the endoscopy procedure with malignancy or inflammatory bowel disease (IBD); and those who were hospitalized.

\section{Statistical Analysis}

A review of the literature [7] suggested an approximately $2 \%$ incidence of endoscopy in the general population, with our internal pilot study suggesting a $6 \%$ incidence of endoscopy in patients with VTE.

The number of occurrences of endoscopy in cases and controls was examined. The first analysis compared the difference between all cases and controls. Nine control subjects were found to have had a prior diagnosis of VTE, so these and their corresponding cases were removed from the analysis.

The second analysis excluded all patients with a new or previous diagnosis of inflammatory bowel disease or malignancy and their corresponding controls. An additional analysis was performed excluding only hospitalized patients and their corresponding controls. Due to the paired nature of the data (due to the matched design), the difference in endoscopy occurrence between cases and controls was examined using the McNemar test. The occurrence of VTE in patients undergoing endoscopy relative to those not undergoing endoscopy was quantified using odds ratios. An examination of the data suggested that there were a sufficient number of colonoscopy and gastroscopy procedures to be considered as separate categories for the purposes of analysis. The numbers of patients undergoing flexible sigmoidoscopy and ERCP were too small to allow separate analysis. To allow for the matched design, conditional logistic regression was used for the analysis to examine the association between endoscopy and the likelihood of VTE.

The analysis was performed using the Stata (version 12.1) statistical package.

\section{Results}

$\nabla$

\section{Patient Characteristics}

From January 2009 to December 2011 we identified 445 patients on the East Kent University Hospitals VTE register. Nine controls (and their respective cases) were excluded, having been previously diagnosed with VTE.

In the first analysis 436 were paired with the same number of age- and sex-matched controls who attended the outpatients department during the same period. Of these 436 patients, $57.7 \%$ were female and $42.3 \%$ were male. The median age at which patients developed VTE was 68 years.

As shown in Table 1, 45/436 in the VTE group had attended for endoscopy in the preceding 3 months while 14/436 had attended for endoscopy in the control group ( $10.3 \%$ vs $3.2 \%$ ). The odds ratio for developing VTE following endoscopy was 3.58 (95\% CI 1.86 - 7.46; $P<0.001$ ).

- Table 2 shows the outcome for the incidence of VTE when hospitalized patients $(n=10)$ and those with a new or previous diagnosis of IBD or malignancy (17) were excluded from the analysis. In this group, the odds of VTE were almost twice as large for pa-

\begin{tabular}{|llllll|}
\hline Analysis & Group & $\mathbf{n}$ & Endoscopy $\mathbf{n}(\%)$ & Odds ratio (95\% Cl) & P-Value \\
\hline Control group & Controls & 436 & $14(3.2 \%)$ & $3.58(1.86-7.46)$ & $<0.001$ \\
\hline VTE group & Cases & 436 & $45(10.3 \%)$ & & \\
\hline
\end{tabular}

Table 1 Incidence of venous thromboembolism in patients and controls

\begin{tabular}{|llllll|}
\hline Analysis & Group & $\mathbf{n}$ & Endoscopy n (\%) & Odds ratio (95\% Cl) & $\boldsymbol{P}$-Value \\
\hline All subjects & Controls & 409 & $13(3.2 \%)$ & $1.92(0.95-3.85)$ & 0.07 \\
\hline & Cases & 409 & $24(5.9 \%)$ & & \\
\hline
\end{tabular}

Table 2 Incidence of venous thromboembolism in patients and controls excluding hospitalized patients and those with inflammatory bowel disease or malignancy 


\begin{tabular}{|llllll|}
\hline Analysis & Group & $\mathbf{n}$ & Endoscopy $\mathbf{n}(\%)$ & Odds ratio (95\% Cl) & P-Value \\
\hline All subjects & Controls & 426 & $13(3.1 \%)$ & $2.92(1.51-5.62)$ & 0.001 \\
\hline & Cases & 426 & $36(8.5 \%)$ & & \\
\hline
\end{tabular}

Table 3 Incidence of venous thrombosis in patients and controls excluding only those who were inpatients

\begin{tabular}{lccll|}
$\begin{array}{l}\text { Endoscopic } \\
\text { procedure }\end{array}$ & Controls n (\%) & Cases $\mathbf{n}(\%)$ & Odds ratio (95\% Cl) & $\begin{array}{l}\text { P-Value } \\
\text { versus none }\end{array}$ \\
\hline None & $401(96.9 \%)$ & $385(94.1 \%)$ & 1 & - \\
\hline $\begin{array}{l}\text { Colonoscopy } \\
\text { Esophagogastro- }\end{array}$ & $5(1.2 \%)$ & $8(1.9 \%)$ & $1.6(0.52-4.89)$ & 0.41 \\
duodenoscopy & $5(1.2 \%)$ & $13(3.1 \%)$ & $2.62(0.93-7.39)$ & 0.07 \\
\hline Others & $3(0.7 \%)$ & $3(0.7 \%)$ & $1.17(0.23-5.96)$ & 0.85 \\
\hline
\end{tabular}

Table 4 Association between type of endoscopic procedure and venous thromboembolism.

Inpatients, patients with irritable bowel disease or malignancy, and those controls (and respective cases) with a past history of venous thromboembolism were excluded from the analysis.

tients undergoing endoscopy as for controls, but this did not reach statistical significance.

- Table 3 shows the incidence of VTE when only hospitalized patients were excluded from the analysis (as many of the patients with IBD or malignancy were diagnosed at the time of the endoscopy procedure). This analysis suggested strong evidence that endoscopy was more common in cases than in control subjects, with $8.5 \%$ of cases and $3.1 \%$ of control undergoing a procedure. The odds of VTE were nearly three times as large for this outpatient cohort undergoing endoscopy than for controls (odds ratio with $95 \% \mathrm{CI}$ : $2.92[1.51-5.62] P=0.001$ ).

\section{Association between VTE and Different Types of Endoscopy}

The number of patients in the VTE cohort who had undergone an endoscopy procedure in the 3 months prior to the diagnosis is shown in Table 4 (which excludes controls with a past history of VTE, inpatients, those with IBD or malignancy, and matched controls). Overall, $94.1 \%$ (385) of patients in the VTE group and $96.9 \%$ in the control group did not have endoscopy. Thirteen patients (3.1\%) underwent a gastroscopy compared with 5 (1.2\%) in the control group (odds ratio 2.62 [0.93-7.39], $P=0.07$ ). Eight (1.9\%) had a colonoscopy in the VTE group compared with 5 $(1.2 \%)$ in the control group (odds ratio $1.6[0.52-4.89] P=0.41$ ).

\section{Timing of VTE after Endoscopy and Diagnosis at Endoscopy}

A total of $9 / 45$ patients (20\%) developed VTE within 7 days of the endoscopy procedure, 9/45 (20\%) between 8 and 30 days, and $27 / 45$ (60\%) later than 30 days after the procedure. Of the 9 patients who developed VTE within 7 days, 7 had been admitted to hospital for their endoscopic procedure. Seventeen out of 45 patients (37.7\%) were confirmed to have either malignancy or IBD, the majority diagnosed at the time of the endoscopy procedure.

\section{Discussion \\ $\nabla$}

Patients undergoing endoscopy had a 3.6-fold increased risk of VTE compared with controls. This risk is comparable to that of women undergoing day surgery (4-fold risk) but lower than patients undergoing inpatient surgery (40-fold). [8-10] In our cohort, the increased risk was mainly the result of recognized risk factors such as inpatient admission or the presence of IBD or malignancy.

One explanation for these findings may be that endoscopy procedures often require patients to remain in the same position for prolonged periods with the use of intravenous sedation. In addition, the increased intraabdominal pressure created by air insufflation during endoscopy may cause venous pooling in the legs, via vessel wall damage, as suggested by animal studies of pneumoperitoneum. [11] Elderly patients are more susceptible to dehydration following bowel preparation, [12] and this might increase their risk of developing VTE.

Although bowel preparation and duration and type of procedure might be expected to influence the risk of VTE associated with endoscopy, when patients with recognized risk factors were removed from the analysis, no statistical difference was noted between those undergoing either a gastroscopy or colonoscopy procedure and risk of VTE. The number of patients with VTE in the ERCP and flexible sigmoidoscopy ("others") category was insufficient to draw any conclusions. In the overall patient group, $40 \%$ developed their VTE within 30 days of endoscopy, and in half of these the VTE developed within 7 days.

Larger studies may highlight whether the type of endoscopic procedure or diagnosis may alter this risk.

A prospective cohort study on women reported that incidence rates for developing VTE in the first 6 weeks after surgery were more than 100-fold following inpatient surgery and 10-fold following day case surgery. [13] The association between VTE developing after an endoscopic procedure (all attendees) is lower than that quoted for surgical patients, but is nonetheless greater than for the outpatient attending control group.

Just over a third of patients who developed VTE either had IBD or malignancy as their endoscopic diagnosis. This finding was not statistically significant but was in line with other studies which confirmed IBD and malignancy as risk factors for developing VTE. An epidemiological review reported IBD as an independent risk factor for VTE, with this cohort reported as having a 1.5 - to 3.5-fold increased risk of developing VTE. [3] Malignancy is associated with increased risk of VTE and the development of such reduced survival in patients with either local or regional disease. [4]

A recent study reported that $10 \%$ of patients developed deep vein thrombosis following endoscopic submucosal dissection of large sessile polyps. [10] This prospective study assessed the risk of VTE only after therapeutic endoscopy. To our knowledge this is 
the first study that has demonstrated an increased risk of VTE in patients attending for endoscopic procedures and highlights the need to consider VTE risk in lengthy therapeutic procedures and-with the evidence from our present study-to extend this to inpatients and those with IBD or a gastrointestinal malignancy. The present study has confirmed that $10 \%$ of patients with confirmed VTE over a 3-year period in our hospital trust had undergone an endoscopy procedure in the 3 months preceding diagnosis, compared with $3 \%$ in the control group $(P<0.001)$. Once inpatients and those with a past history of IBD or malignancy were excluded, there was no significant difference between cases and controls with respect to VTE risk.

\section{Implications for Practice}

The occurrence of VTE, a clinically serious condition, is thought to be rare after endoscopy because early ambulation is usually allowed, whereas mobilization after surgical procedures is somewhat slower. The present study has shown that there is a 3.6fold increased risk of VTE following endoscopy compared with the risk in controls attending for an outpatient appointment. The increased risk is largely in the cohort of patients with recognized risk factors. Our results suggest that a greater awareness of the need for VTE prophylaxis in patients attending for endoscopy procedures may be required.

\section{Limitations}

$\nabla$

This study was a single-center retrospective study. Although the sample size was adequate to identify a statistical difference in the risk of VTE following endoscopy, it was not large enough to establish a statistically significant relationship between the type of endoscopic procedure and risk of developing VTE.

\section{Competing interests: None}

\section{Acknowledgments}

$\nabla$

We thank Dr Paul Bassett, Statsconsultancy Ltd., UK, for providing statistical advice, and Dr Paul Kiwanuka-Musoke for his help with data collection.

\section{References}

1 Cohen AT, Agnelli G, Anderson FA et al. Venous thromboembolism (VTE) in Europe. The number of VTE events and associated morbidity and mortality. Thromb Haemost 2007; 98: 756-764

2 Geerts WH, Bergqvist D, Pineo GF et al. Prevention of venous thromboembolism: American College of Chest Physicians Evidence-Based Clinical Practice Guidelines (8th Edition). Chest 2008; 133: 381 - 453

3 Murthy SK, Nguyen GC. Venous thromboembolism in inflammatory bowel disease: an epidemiological review. Am J Gastroenterol 2011; 106: $713-718$

4 Alcalay A, Wun T, Khatri Vet al. Venous thromboembolism in patients with colorectal cancer: incidence and effect on survival. J Clin Oncol 2006; $24: 1112-1118$

5 UK Department of Health. The standard NHS contracts for acute hospital, mental health, community and ambulance services and support guidance. The National Archives; London, UK: 2010

6 UK Department of Health. Commissioning for quality and innovation 2013/2014 Guidance. National Health Service; London, UK: 2013

7 Barrison IG, Bramble MG, Wilkinson $M$ et al. Provision of endoscopy related services in district general hospitals. British Society of Gastroenterology; 2001

8 Parkin L, Sweetland S, Balkwill A et al. Body mass index, surgery, and risk of venous thromboembolism in middle-aged women: a cohort study. Circulation 2012; 125: 1897 - 1904

9 Buchberg B, Masoomi H, Lusby K et al. Incidence and risk factors of venous thromboembolism in colorectal surgery: does laparoscopy impart an advantage? Arch Surg 2011; 146: 739-743

10 Kusunoki MK, Miyake K, Shindo T et al. The incidence of deep vein thrombosis in Japanese patients undergoing endoscopic submucosal dissection. Gastrointest Endosc 2011; 74: 798-804

11 Jorgensen JO, Gillies RB, Lalak NJ et al. Lower limb venous hemodynamics during laparoscopy: an animal study. Surg Laparosc Endosc 1994; 4: $32-35$

12 Seinelä L, Pehkonen E, Laasanen T et al. Bowel preparation for colonoscopy in very old patients: a randomized prospective trial comparing oral sodium phosphate and polyethylene glycol electrolyte lavage solution. Scand J Gastroenterol 2003; 38: 216-220

13 Sweetland S, Green J, Liu B et al. Duration and magnitude of the postoperative risk of venous thromboembolism in middle aged women: prospective cohort study. BMJ 2009; 339: b4583 